\title{
Multiyear Transmission Expansion Planning Using Ordinal Optimization
}

\author{
Min Xie, Jin Zhong, Member, IEEE, and Felix F. Wu, Fellow, IEEE
}

\begin{abstract}
The increasing complexity of the transmission expansion planning problem in the restructured industry makes simulation the only viable means to evaluate and compare the performances of different plans. Ordinal optimization is an approach suitable for solving the simulation-based multiyear transmission expansion planning problem. It uses crude models and rough estimates to derive a small set of plans for which simulations are necessary and worthwhile to find good enough solutions. In the end, reasonable solutions are obtained with drastically reduced computational burden.
\end{abstract}

Index Terms-Multiyear transmission expansion, ordinal optimization, transmission planning.

\section{INTRODUCTION}

$\mathbf{T}$ RANSMISSION EXPANSION PLANNING (TEP) aims at strengthening an existing transmission network to serve power producers and customers in an optimal way. Due to the large-scale nature of a transmission system and its inherent complexities, TEP has always been a complex problem. It has been formulated as a large-scale mixed-integer nonlinear optimization problem. Various optimization techniques have been used to solve the problem, such as linear programming [1], [2], dynamic programming, nonlinear programming [3], [4], and mixed-integer programming [5]-[8]. At the same time, various divide-and-conquer strategies such as Benders decomposition [9]-[11], hierarchical decomposition, and branch-and-bound algorithm have also been applied to solve large-scale TEP problems. A more complete literature survey can be found in [12].

Industry restructuring in recent years has resulted in the separation of generation and transmission systems and the introduction of competitive electricity markets. A comprehensive methodology, called the Transmission Economic Assessment Methodology (TEAM), was developed by the California ISO and London Economics to evaluate the economic benefits of transmission expansion [13]. TEAM advocates the use of simulations for a large number of scenarios.

Manuscript received October 9, 2006; revised July 31, 2007. This work was supported by the Research Grant Council of Hong Kong under Grant 7197/06E, 7174/04E, and the National Key Basic Research Project of China under Grant 2004CB217900. Paper no. TPWRS-00697-2006.

M. Xie is with the School of Electric Power, South China University of Technology, Guangzhou, China (e-mail: minxie@scut.edu.cn).

J. Zhong and F. F. Wu are with the Department of Electrical and Electronic Engineering, The University of Hong Kong, Hong Kong, China (e-mail: jzhong@eee.hku.hk; ffwu@eee.hku.hk).

Color versions of one or more of the figures in this paper are available online at http://ieeexplore.ieee.org.

Digital Object Identifier 10.1109/TPWRS.2007.907160
The economic impacts of alternative transmission enhancement schemes are different for different market participants. The participants of an electricity market include independent power producers, large customers, transmission network owners, and the independent system operator. The interests of different parties vary a great deal. Furthermore, other factors, such as uncertainties in generation patterns, transmission congestions, and regulatory policy changes, need to be considered in the evaluation. Transmission expansion planning in the restructured industry has become much more complicated than before [14]. No simple mathematical model can capture all the major factors in the transmission expansion planning. Computer simulation, in particular Monte Carlo simulation, has become the only viable approach for assessing alternative plans for transmission expansion.

A large number of scenarios and operating conditions, as well as uncertainties regarding contracts and bidding, must be considered in planning. Simulation-based transmission planning of such a stochastic system for practical large networks will require computational resources that are commonly unavailable.

If, on the other hand, analytical approaches can be used to complement the simulation-based search methods so that the search for optimum performance can be narrowed down to a set of good enough solutions, then the computational requirements may be manageable. Ordinal Optimization (OO) [15] is a method that provides a theoretical foundation for such an approach.

In Section II, a TEP problem is described. Ordinal optimization theory is introduced in Section III. In Section IV, ordinal optimization theory is applied to solve the TEP problem. In Section V, numerical examples are given to illustrate the approach. Conclusions are drawn in Section VI.

\section{TRANSMission EXPANSION PlanNing PROBLEM}

We assume that there is an entity, which may be the transmission company, the independent system operator (ISO), or the regional transmission organization (RTO), who is responsible for planning the expansion of the transmission network (i.e., when and where to install new lines, capacities and types, etc.). The economic effects of TEP on various market players are different. Transmission owners are concerned about their investment returns; generation companies are about congestion rents affecting their profits; the system operator is about congestion revenues; and the consumers are about electricity prices after network enhancement. On the other hand, societal outage costs may very well be reduced after adding line capacities in the network. The magnitudes of the economic effects on generators, consumers, the system operator, and the society depend on system operating 
conditions that change from moment to moment. The benefit of an expansion scheme to an individual participant may take several years to realize. To simulate the effects of multiple years of an expansion plan on market players, an hourly-based dispatch model is more or less necessary for the whole expansion time horizon.

Industry restructuring is an ongoing process in many parts of the world. Its initial focus has been on the competitive market for generation. Transmission systems remain largely regulated, and the rules and regulations for transmission expansion are mostly unsettled. The obligations and responsibilities of the participants are not fully defined, except that some general characteristics can be detected. The TEP has become an optimization problem whose variables are strongly stochastic and lumpy (discrete), with multiple participants having different objectives. Traditional optimization formulations and techniques are no longer appropriate. In this paper, we propose the application of ordinal optimization to the TEP problem. The development of $\mathrm{OO}$ is motivated by the complexities of large-scale, stochastic, discrete-event nonlinear dynamic systems, such as manufacturing systems, whose performance can only be evaluated by way of computer simulations.

Because the TEP problem is not fully standardized and specified, we will not attempt to give a definitive algorithmic solution to the problem. Instead, our goal is to demonstrate that the OO approach is viable. Therefore, our formulation of the TEP problem and its solution algorithm are for illustration purposes only. For that purpose and for the ease of exposition and understanding, the classical TEP formulation which has been commonly used in the past decades is used in this paper. The OO approach can be adopted by the planners to a formulation that incorporates the issues and considerations relevant to individual systems and to the development of models and performance indexes that the $\mathrm{OO}$ approach requires.

\section{A. Classical TEP Model}

For simplicity, we use the classical optimization model in this paper to represent the multiyear TEP problem. We assume that the objective function of the exact TEP model is to minimize the total cost, which is formulated as (1)

$$
\min T=I+G+L
$$

where $T$ represents total cost; $I$ represents the investment cost; $G$ represents production cost under the optimal dispatching condition; and $L$ represents the cost of loss of load as a result of contingencies.

We assume that the expanded line capacities are added yearly in a Y-year span and that the right-of-ways planned and authorized for building new lines are already specified. The transmission planner has the option to decide on which right-of-ways to use in order to build new lines and their capacities. Each combination of the lines built in one year is called a transmission expansion scheme for that year. The annual planning schemes over the span of Y-years are illustrated in Table I. Assume there are $M$ right-of-ways authorized for building new lines, the number of lines built on the right-of-way $m(m=1, \ldots, M)$ in the year $y(y=1, \ldots, Y)$ is Line $_{m y}$. Each column in the table
TABLE I

PlanNing SCHEMES IN Y-YeAR TRANSMISSION EXPANSION PLANNING

\begin{tabular}{|c|cclc|}
\hline Right-of-way $m$ & Year 1 & Year 2 & $\ldots \ldots$ & Year Y \\
\hline Right-of-way 1 & Line $_{11}$ & Line $_{12}$ & $\ldots \ldots$ & Line $_{1 Y}$ \\
Right-of-way 2 & Line $_{21}$ & Line $_{22}$ & $\ldots \ldots$ & Line $_{2 Y}$ \\
$\ldots \ldots$. & $\ldots \ldots$ & $\ldots \ldots$ & $\ldots \ldots$ & $\ldots \ldots$ \\
Right-of-way $M$ & Line $_{\mathrm{M} 1}$ & Line $_{\mathrm{M} 2}$ & $\ldots \ldots$ & Line $_{\mathrm{MY}}$ \\
\hline
\end{tabular}

represents the expansion scheme of the year. The whole table represents the expansion scheme of the whole planning span.

1) Investment Cost: The investment cost is calculated as the total expansion investment over the planning span. It is formulated as (2)

$$
\begin{aligned}
I & =\sum_{y=1}^{Y}\left(\frac{I^{y}}{(1+\gamma)^{y-1}}\right) \\
I^{y} & =\sum_{m=1}^{M} I_{m}^{y}
\end{aligned}
$$

where symbol $I_{m}^{y}$ represents the expansion investment on the $m^{\text {th }}$ right-of-way in year $y ; I^{y}$ is the investment cost in year $y ; \gamma$ is the discount rate; and $I$ is the investment value corresponding to the beginning year of the investment. The Net Present Value (NPV) approach is used here.

2) Production Cost: The commonly used quadratic function (3) is used to represent the production cost function of a generator $i$. The optimal hourly production cost $G_{h}$ can be obtained by minimizing the total generation cost for each hour $h=1, \ldots, 8760$, subject to the power balance (dc load flow) and other operating constraints.

$$
\begin{gathered}
G_{h}=\min \sum_{i}\left(a_{i} g_{i}^{2}+b_{i} g_{i}+c_{i}\right) \\
\text { s.t. } g_{i}-d_{i}=\sum_{j=1}^{N} B_{i j} \theta_{i} \\
\sum_{i} g_{i}=D_{h} \\
\underline{l_{i j}} \leq B_{i j}\left(\theta_{i}-\theta_{j}\right) \leq \overline{l_{i j}} \\
\quad \underline{g_{i}} \leq g_{i} \leq \overline{g_{i}} \\
i, j=1, \ldots N
\end{gathered}
$$

where $g_{i}$ represents the real power generation of the generator on bus $i ; a_{i}, b_{i}$, and $c_{i}$ are the constant coefficients of power generation; $D_{h}$ is the total load in hour $h ; B_{i j}$ represents the susceptance between node $i$ and $j ; \theta_{i}$ represents the phase angle of node $i ; \overline{l_{i j}}$ and $l_{i j}$ represent the upper and lower limits of line $i j ; \overline{g_{i}}$ and $\underline{g_{i}}$ represent the upper and lower limits of $g_{i}$; and $N$ is the total number of nodes.

For year $y$, the total production cost $G^{y}$ is the sum of $G_{h}$ for $8760 \mathrm{~h}$

$$
G^{y}=\sum_{h=1}^{8760} G_{h} .
$$


The NPV of the production cost for the whole planning span can be represented as (5)

$$
G=\sum_{y=1}^{Y}\left(\frac{G^{y}}{(1+\gamma)^{y-1}}\right) .
$$

3) Cost of Loss of Load: In the abnormal operation conditions, a good network configuration may help to avoid load shedding. Providing a reliable network under system contingencies is one of the goals of transmission expansion planning. The dispatch modes are different for different transmission network configurations.

The loss of load cost (LOLC) depends on the cost of load shedding due to a system contingency. The LOLC can be calculated using one of the three loss-of-cost functions: exponential, quadratic, and hyperbolic functions [16]. In this paper, the quadratic form $\varphi(\bullet)$ as in (6) is used to represent LOLC

$$
\varphi_{n, h}(\Delta)=\frac{4 \xi}{\left(D_{h}^{n}\right)^{2}} \Delta^{2}
$$

where $\varphi_{n, h}$ represents LOLC at node $n$ in hour $h ; \Delta$ represents the amount of lost load, $\Delta=\left(D_{h}^{n}-d^{n}\right)$, where $D_{h}^{n}$ is the load at node $n$ in hour $h$; and $d^{n}$ is the load at node $n$ after the contingency. $\xi$ is a rough-estimated cost assuming that half of the total load is lost. In Europe, $\xi$ is usually assumed to be 30-60 times the regular electricity price [16].

The value of LOLC caused by the contingency in hour $h, L_{h}$, can be formulated as (7)

$$
L_{h}=\sum_{n=1}^{N} \varphi_{n, h}\left(D_{h}^{n}-d^{n}\right) .
$$

For the given probabilities of all contingencies, we can obtain the expected hourly LOLC for all contingencies considered. The expected hourly LOLC, $E L_{h}$ is formulated as (8)

$$
E L_{h}=\sum_{k=1}^{K} p_{k} L_{h, k}
$$

where $K$ is the size of the contingency set considered; and $p_{k}$ is the probability of contingency $k$. The yearly LOLC can be formulated as the summation of $E L_{h}$, as shown in (9)

$$
L^{y}=\sum_{h=1}^{8760} E L_{h}
$$

The total LOLC over the whole planning horizon can be obtained by (10)

$$
L=\sum_{y=1}^{Y}\left(\frac{L^{y}}{(1+\gamma)^{y-1}}\right) .
$$

To solve the proposed TEP model, (1)-(10), both the normal and abnormal operation modes of all hours of the whole planning span need to be calculated. Obtaining the exact solution for a multiyear TEP problem will result in a huge computational burden.

\section{ORDINAL OPTIMIZATION}

The ordinal optimization theory developed by Ho et al. [14], [17]-[25] is for solving simulation-based complex optimization problems. It has recently been applied to many areas in power systems such as optimal power flow (OPF) with discrete control [26] and bidding strategies of power suppliers in markets [27]. In this paper, the theory is applied to solve the multiyear transmission expansion planning problem.

The goal of ordinal optimization is to find good enough solutions for a complex optimization problem. A good enough subset $G(\Theta)$ is the subset consisting of the top best solutions, say, the top 5\% in the solution space. However, it is difficult to find the subset $\mathrm{G}$ for a simulation-based problem unless all the solutions in the solution space are calculated and compared. The OO method uses rough estimates from a crude model to rank the solutions. However, even with the use of a crude model, estimation of performance values for all solutions of a large solution space $\Theta$ may not be computationally feasible. Ordinal optimization theory uses a representative set with $\mathrm{N}$ samples, $\Theta^{\mathrm{N}}$, to represent the original solution space $\Theta$. If the elements of the representative set are randomly selected, the probability of an event where at least one of the $\mathrm{N}$ samples will fall within the top 5\% of the whole solution space is

$$
\mathrm{P}=1-(1-5 \%)^{\mathrm{N}} .
$$

If $\mathrm{N}=1000$, then the probability that the top $5 \%$ good enough solutions are not in the $\mathrm{N}=1000$ samples is $\mathrm{P}=0.95^{1000}=$ $5.29 \times 10^{-23} \approx 0$, which is extremely small (of the order $10^{-23}$ ). In this paper, we will use $\mathrm{N}=1000$ samples to represent the solution space $\Theta$. However, calculating all $\mathrm{N}=1000$ accurate solutions by computer simulations is still a formidable task. The goal of ordinal optimization is to reduce the number of necessary but computationally costly simulations.

\section{A. Good Enough Subset and Selected Subset}

Within the defined finite solution space $\Theta^{\mathrm{N}}\left(\left|\Theta^{\mathrm{N}}\right|=1000\right)$, the number of top $n \%$ solutions is $g=N * n \%$. The top $g$ solutions compose the "good enough" subset $\mathrm{G}$ of $\Theta^{\mathrm{N}}$. In the later sections of this paper, $g$ is selected to be $1000 * 5 \%=50$.

The multiyear TEP problem proposed in Section II can symbolically be represented as finding the minimum performance value $\mathrm{T}$ among all possible expansion schemes

$$
\min T=T(x)
$$

where the variable $x$ corresponds to a set of line expansion schemes. For example, building certain lines on the approved right-of-ways in each year of the Y-year span is referred to as an expansion scheme. Assuming there are 1000 potential expansion schemes $x_{1}, \ldots, x_{1000}$, then a high quality solution could be found by calculating the performance values for 1000 schemes, $T_{1}, \ldots, T_{1000}$, and choosing for the best one. Such an exhaustive search method is what we want to avoid. Instead, we reduce the search space to a small selected subset $\mathrm{S}\left(\Theta^{\mathrm{N}}\right)$ and perform simulations within the selected subset.

The key to the determination of a selected subset is to be sure that the selected subset intersects with the "good enough" 


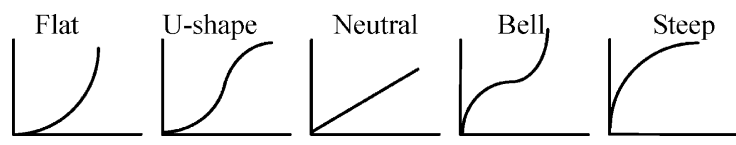

Fig. 1. Five types of OPCs.

subset. The selected subset may be determined by using certain fast evaluation methods, such as mathematical algorithms, heuristics, etc., based on the crude approximate models of the system.

For example, we may first use a crude model to obtain a rough estimate of the performance values $T_{1}^{\prime}, \ldots, T_{1000}^{\prime}$ for the potential expansion schemes $x_{1}, \ldots, x_{1000}$ in problem (11). The rough evaluation should take much less time than the accurate calculation. Thus, the top 50 schemes found by the crude model may not be the same as (i.e., not aligned to) the top 50 schemes (subset $\mathrm{G}$ ) obtained from the accurate model. However, if we select enough schemes (subset $\mathrm{S}$ ) from the rough estimates, the selected subset $\mathrm{S}$ will have a high probability of overlapping with the elements in $\mathrm{G}$. The number of overlapped elements with $\mathrm{G}$ is called the alignment level $k$. Our goal is to find a subset $\mathrm{S}$ including at least $k$ elements of $\mathrm{G}$. The probability of this event is called the alignment probability $p$.

For a given alignment probability $p$ and the alignment level $k$, the size of the selected subset $\mathrm{S}$ is determined by the requirement that the probability that $S$ overlaps the good enough subset $G$ with at least $k$ elements is greater than $p$

$$
\operatorname{Prob}\{|G \cap S| \geq k\} \geq p \text {. }
$$

Obviously, the determination of the size of the selected subset is dependent on the nature of the underlying optimization problem. The ordinal optimization theory broadly divides the optimization problems into several classes. The classification is accomplished by way of constructing an ordered performance curve to be introduced below.

\section{B. Ordered Performance Curve}

The ordered performance curve (OPC) may be constructed based on the estimated performance values obtained by the crude model. The 1000 estimated performance values are arranged in an ascending order (for minimization problem). The $\mathrm{X}$ axis of the resulting plot is the scheme labels; whereas the $\mathrm{Y}$ axis represents the (estimated) performance values. The shape of an OPC determines the nature of the underlying optimization problem.

The shapes of OPC curves can be broadly categorized into five classes: flat, u-shape, neutral, bell, and steep, as shown in Fig. 1 [14], [17].

For a minimization problem, a smaller performance value means a good scheme, and a bigger performance value means a bad scheme. For a problem, if more small-value schemes are found, then the problem has more good schemes. In Fig. 1, the problem with a flat-shape OPC has more good schemes. The five OPC curves in the figure represent five classes of optimization problems: 1) flat-many good schemes, 2) U-shape_many good and bad schemes, 3) neutral—good and
TABLE II

Size of SElected Subset for Five OPC-BASEd PROBLEMS

\begin{tabular}{|c|c|c|c|c|c|}
\hline \multirow{2}{*}{$\mathrm{g}=50$} & \multicolumn{5}{|c|}{ Ordered Performance Curve (OPC) shape } \\
\cline { 2 - 6 } & Flat & U-shape & Neutral & Bell & Steep \\
\hline $\mathrm{k}=1$ & 37 & 25 & 22 & 12 & 11 \\
\hline $\mathrm{k}=2$ & 63 & 41 & 35 & 15 & 13 \\
\hline $\mathrm{k}=3$ & 88 & 57 & 48 & 21 & 14 \\
\hline $\mathrm{k}=4$ & 113 & 73 & 61 & 29 & 16 \\
\hline $\mathrm{k}=5$ & 136 & 89 & 71 & 39 & 19 \\
\hline
\end{tabular}

\begin{tabular}{|l|c|c|c|c|c|}
\hline \multirow{2}{*}{$\mathrm{g}=10$} & \multicolumn{5}{|c|}{ Ordered Performance Curve (OPC) shape } \\
\cline { 2 - 6 } & Flat & U-shape & Neutral & Bell & Steep \\
\hline $\mathrm{k}=1$ & 219 & 153 & 125 & 45 & 31 \\
\hline
\end{tabular}

bad schemes equally distributed, 4) bell—many intermediate schemes, and 5) steep-many bad schemes.

\section{Size of Selected Subset}

In [17], through extensive simulations and statistical analysis, a formula is derived to relate the size of the selected subset $\mathrm{S}$ to i) the shape of the OPC, ii) the size of good enough subset G, iii) the alignment level $k$, iv) the alignment probability $p$, and v) the error bound $w$ between the performance values from the crude model and the accurate model.

Assume the requirements for our TEP optimization problem are

- $\quad$ size of representative set $\mathrm{N}=1000$;

- "good enough" subset $\mathrm{G}$ is defined as the top $5 \%$ solutions of $\Theta^{\mathrm{N}}$; thus, $\mathrm{g}=\mathrm{N} * \mathrm{n} \%=1000 * 5 \%=50$;

- alignment level of $\mathrm{G} \cap \mathrm{S}$ is $k=1,2,3$, 4, or 5;

- alignment probability of $\mathrm{G} \cap \mathrm{S}$ is $p=95 \%$.

Assuming the error bound is 0.5 , the size of the selected subset for the optimization problems with the five different OPC shapes are calculated based on the formula provided in [17], and the results are tabulated in Table II. The size of the selected subset for the case $g=10$ and $k=1$ is also given in Table II for comparison.

From Table II, we find that, for an optimization problem with a Bell shape OPC curve, the size of the selected subset is $s=12$ (for $k=1$ ). This means that, after a rough estimation, if we pick the best 12 schemes from the rough estimation to run the exact evaluations, there is a $95 \%$ probability that at least one scheme $(k=1)$ out of the 12 will fall in the "good enough" subset $\mathrm{G}$. If alignment level $k$ is set to be 2 , then 15 schemes need to be calculated for exact evaluations to guarantee that at least two schemes will fall in the good enough subset $\mathrm{G}$ with the probability of $95 \%$.

Note from the rows of Table II that the size of the selected subset decreases from a Flat shape to a Steep shape, that is, an optimization problem with a Flat OPC shape requires more selected schemes than a problem with a Steep OPC curve. This is because for a Flat shape OPC, there are many schemes whose performance values based on the crude model are more or less equally good (small). The exact performance value of each individual scheme is the estimated value plus the error in the estimation. The error terms are unknown. Adding such error terms will change the order of the ranking of the schemes that are flat (more or less the same performance) significantly. Therefore, 
more schemes need to be selected in order to capture enough good enough schemes.

On the other hand, if the OPC is Steep, although fewer schemes out of the 1000 are needed for an exact calculation, the quality of the 1000 samples may be lower. In this case, it may be prudent to increase the initial sample from 1000 to a larger number in order to capture more "good enough" schemes.

\section{CRUde Model For TEP PROBlem}

In this section, we propose crude models for the quick estimation of investment cost, production cost, and loss of load cost to be used in the ordinal optimization approach.

\section{A. Investment Cost}

The exact formula (2) for the investment cost does not require much computation effort; therefore, it can be used directly.

\section{B. Crude Model for Production Cost}

The evaluation of production cost given in (5) for a span of Y-years requires carrying out simulations (solving $\mathrm{OPF}$ ) for $\mathrm{Y} * 8760$ periods, which constitutes a considerable computational burden. A crude model is needed for the rough estimation of production cost. For example, a possible crude model may use a seasonal typical-day dispatch mode and production cost to represent all the days in the season. The simulation times can thus be reduced from $\mathrm{Y} * 8760$ to $\mathrm{Y} * 4 * 24=\mathrm{Y} * 96$, almost a 100 -fold reduction. In this paper, the symbol` is used to represent the rough estimate of the value from the crude model. The estimated production cost from the crude model can be expressed as (13)

$$
\hat{G}=\sum_{y=1}^{Y}\left(\frac{\hat{G}^{y}}{(1+\gamma)^{y-1}}\right) .
$$

\section{Crude Model for Loss of Load Cost}

A piecewise linear approximation can be used to give a rough estimate of the cost of loss of load, as expressed in (14)

$$
\hat{L}=\sum_{y=1}^{Y}\left(\frac{\hat{L}^{y}}{(1+\gamma)^{y-1}}\right) .
$$

In the approximation, a few load points between peak load and off-peak load are chosen. For example, we can select the base load level and the medium load level. The selected load points divide the load curve into several pieces. By calculating the LOLC of the selected load levels, we can use the linear approximation to roughly calculate the LOLC for all load levels.

\section{Crude Model of TEP Cost}

By combining (2), (13), and (14), a rough estimate of the total cost of the TEP problem (1) can be obtained as in (15)

$$
\min \hat{T}=I+\hat{G}+\hat{L} .
$$

The rough estimates obtained from (15), of course, are different from the accurate values obtained from the accurate simulation
TABLE III

ORIGINAL AND MAXIMUM TARGET NUMBER OF LINES

\begin{tabular}{c|ccccccccccc}
\hline Right-of-way & 1 & 2 & 3 & 4 & 5 & 6 & 7 & 8 & 9 & 10 & 11 \\
\hline original no. of lines & 1 & 1 & 1 & 0 & 1 & 1 & 0 & 1 & 0 & 0 & 0 \\
max target no. of lines & 4 & 4 & 4 & 4 & 4 & 4 & 4 & 4 & 4 & 4 & 4 \\
\hline
\end{tabular}

models (1)-(10). The difference is the error which can be regarded as a random variable [15]

$$
T=\hat{T}+\text { error }
$$

The probability distribution of the random variable error is usually assumed to follow a uniform distribution $\mu(-w, w)$ in [17]. An estimate of the error bound $w$ can be obtained by performing a calculation of a small number of schemes for both the exact and rough estimates of the performance values. $w$ will be used in the calculation of the size of the selected subset.

\section{ILLUSTRATIVE EXAMPLES}

The six-node Garver system [1] is used to demonstrate the proposed ordinal optimization approach to the multiyear TEP problem. The data of the Garver system are given in the Appendix. There are 11 right-of-ways available for transmission line expansions. The time span for the expansion is assumed to be five years.

The network configuration of the original Garver system and the numbers of the target expansion lines are given in Table III. The index numbers of the right-of-ways in the first row are the same as that in the Appendix.

Assume the annual growth rate of the system peak-load is $20 \%$ and the discount rate is $8 \%$. The $\mathrm{N}-1$ security criterion is used for the contingency analysis to calculate the loss of load cost. The outage probabilities of existing lines are set to be 0.01 , while that of the new lines are assumed to be 0.005 . It is forecasted that, at the end of the planning horizon, the system peak load will reach $1530 \mathrm{MW}$. The load curve of the seasonal typical-day is chosen as the daily load curves for all days in the season.

\section{A. Create the Representative Set $\Theta_{N}$}

There are 746496 possible ways to add new lines to the 11 right-of-ways. For five years, the number comes out to be 3732480 . After eliminating infeasible schemes, the number will still be staggering. As suggested by the ordinal optimization theory, a representative set of 1000 samples may be used to represent the original solution space. The 1000 samples are selected randomly. However, each expansion scheme is tested for each year for feasibility (i.e., to see if it satisfies the yearly peak load requirement without violating operating constraints). If the requirement is not satisfied, the scheme will not be selected as a sample. The procedure is repeated until 1000 feasible samples are selected.

\section{B. Ordered Performance Curve}

Based on the crude model given in (15), the total cost of each sample scheme is calculated roughly. The ordered performance curve can be drawn based on the rough estimates, as shown in 


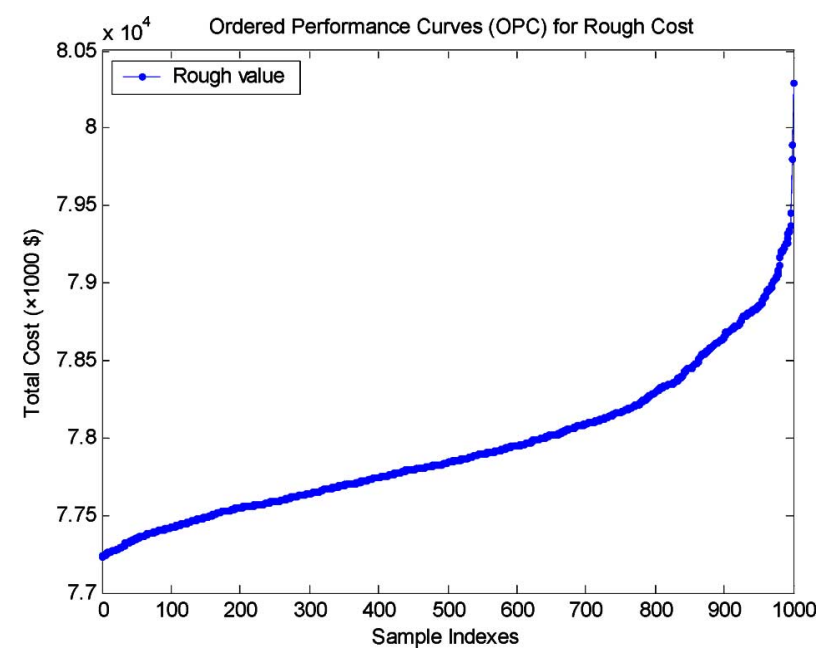

Fig. 2. Ordered performance curve for rough estimation.

Fig. 2. In comparison to the five OPC shapes in Fig. 1, we find that the OPC curve of the proposed TEP problem is a Bell shape curve.

To obtain the error bound $w, 30$ samples are selected for accurate calculations. The standard deviation of the normalized error of the 30 samples is found to be $\sigma=0.1701$. We use $2 \sigma=0.3402$ as the error bound and $w$ as one of the parameters in the determination of the size of selected subset.

\section{Size of Selected Subset}

Based on the requirement listed in Section III-C, suppose we want to have at least $\mathrm{k}=1$ "good enough" solution in the selected subset, we find that the size of selected subset for Bell shape OPC is $s=12$ according to Table II.

\section{D. “Good Enough” Solutions}

The top 12 schemes obtained by (15) are selected as belonging to the selected subset $S$. In Table IV, the index numbers of the schemes in $S$ are listed according to the ascending order of the objective values $\hat{T}$. They are selected from $\mathrm{N}=1000$ samples. After calculating the 12 schemes accurately, it is found that the best solution in the selected set is scheme no. 865 , which is ranked as the eighth by the rough estimation. The "good enough" subset G (top 5\% of $\Theta^{N}$ ) is calculated and listed in Table IV. The squared index numbers with shadows are the schemes that fall in both S and G. As described in (12), there is a $95 \%$ probability that at least one $(k=1)$ element will fall in both $\mathrm{G}$ and $\mathrm{S},(S \cap G)$. In this case, the number of the elements in $S \cap G$ is much higher than the required alignment level $k=1$. The results of this case are much better than expected; all elements in $\mathrm{S}$ fall into $\mathrm{G}$, however, with different ordering in rankings. The cases for $k=2$ and $k=3$ are also calculated and listed in Table IV.

The results of the expansion plans for the three best schemes: no. 865, no. 854, and no. 291, are listed in Table V

For comparison, the case of the top $1 \%$ good enough solutions $(\mathrm{g}=10)$ is calculated and the results are listed in Table VI.

When Table VI is compared with Table IV, the following are found.
TABLE IV

Planning Schemes in the Selected Subset and "Good Enough" Subset (SUBSET G IS TOP $5 \%$ OF $\Theta^{N}$ )

\begin{tabular}{|c|c|}
\hline $\begin{array}{l}\text { Selected subset } \mathrm{S} \\
\quad(k=1, S=12)\end{array}$ & 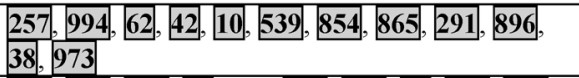 \\
\hline $\begin{array}{l}\text { Good enough set } \mathrm{G} \\
\qquad(g=50)\end{array}$ & $\begin{array}{l}\mathbf{8 6 5}, \mathbf{8 5 4}, \mathbf{2 9 1}, \mathbf{4 2}, 189, \mathbf{9 9 4}, \mathbf{2 5 7}, \mathbf{6 2}, \mathbf{5 3 9}, \mathbf{9 7 3}, \\
\mathbf{1 9 8}, \mathbf{1 0}, 683,656,572,944,768, \mathbf{3 8}, \mathbf{2 4 7}, \mathbf{8 9 6}, 99 \text {, } \\
860,256,450,809,361,111,30,551,166,621, \\
383,440,233,934,442,345,710,643,332,470, \\
246,333,284,351,878,377,476,254,919\end{array}$ \\
\hline
\end{tabular}

\begin{tabular}{|c|c|}
\hline $\begin{array}{l}\text { Selected subset } \mathrm{S} \\
\quad(k=2, S=15)\end{array}$ & 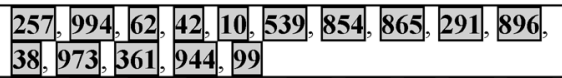 \\
\hline $\begin{array}{l}\text { Good enough set } \mathrm{G} \\
(\mathrm{g}=50)\end{array}$ & $\begin{array}{l}\mathbf{8 6 5}, \mathbf{8 5 4}, \mathbf{2 9 1}, \mathbf{4 2}, 189, \mathbf{9 9 4}, \mathbf{2 5 7}, \mathbf{6 2}, \mathbf{5 3 9}, \mathbf{9 7 3}, \\
\mathbf{1 9 8}, \mathbf{1 0}, 683,656,572, \mathbf{9 4 4}, 768, \mathbf{3 8}, 247, \mathbf{8 9 6}, \\
\mathbf{9 9}, 860,256,450,809, \mathbf{3 6 1}, 111,30,551,166, \\
621,383,440,233,934,442,345,710,643,332, \\
470,246,333,284,351,878,377,476,254,919\end{array}$ \\
\hline
\end{tabular}

\begin{tabular}{|c|c|}
\hline $\begin{array}{l}\text { Selected subset } S \\
\quad(k=3, S=21)\end{array}$ & 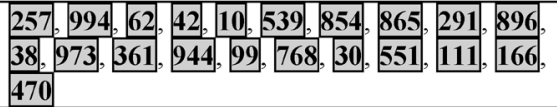 \\
\hline $\begin{array}{l}\text { Good enough set } \mathrm{G} \\
\qquad(\mathrm{g}=50)\end{array}$ & 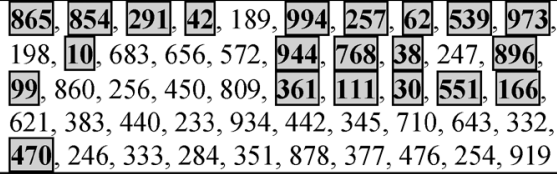 \\
\hline
\end{tabular}

- More schemes are required for an accurate calculation to obtain the top $1 \%$ good enough solutions.

- In Table VI, all top 10 good enough schemes are captured in the selected subset; while the results of Table IV missed the top no. 5 scheme, which has scheme no. 189.

- The results of Table VI $(g=10)$ are more accurate than that of Table IV $(g=50)$ and more accurate calculations are required to obtain the accuracy.

\section{E. Comparison of Rough and Accurate Evaluations}

For each of the 1000 sample expansion schemes, we carry out the accurate evaluation of (1)-(10) based on the exact simulation model. The rough estimated values $\hat{T}$ and the exact values $T$ are plotted in Fig. 3. The sample indexes are ranked according to the ascending order of accurate simulation results (the smooth curve).

The errors between the rough estimates and the exact values are calculated according to the following:

$$
\begin{aligned}
& \text { Error }(\text { in } 10 \mathrm{k} \$) \\
& \quad=\text { Rough total cost }- \text { Exact total cost } \\
& \text { Normalized error }(\%) \\
& \quad=\mid \text { Error }(\text { in } 10 \mathrm{k} \$) \mid / \text { Exact total cost. }
\end{aligned}
$$

Fig. 3 shows that 1 ) the errors arising from the calculations based on the crude model seem to be biased downward, and 2) the errors get larger when the cost is higher. The error bias is attributed to the way the crude model is constructed in which the congestion costs that occur mainly at peak loading were largely uncaptured. The high cost cases are mostly due to the contributions from the loss-of-load costs which are more sensitive to whether exact operating constraints can be satisfied. 
TABLE V

(a) LiNE EXPANSION OF SCHEME No. 865 (UNIT: $10000 \$$ ). (b) LINE EXPANSION OF SCHEME No. 854 (UNIT: 10 000\$). (c) LINE EXPANSION OF SCHEME No. 291 (UNIT: $10000 \$$ )

(a)

\begin{tabular}{ccccccc}
\hline $\begin{array}{c}\text { right-of- } \\
\text { way }\end{array}$ & Year 1 & Year 2 & Year 3 & Year 4 & Year 5 & Total \\
\hline $1(1-2)$ & 1 & 1 & 0 & 1 & 1 & 4 \\
$2(1-4)$ & 1 & 1 & 0 & 0 & 0 & 2 \\
$3(1-5)$ & 1 & 1 & 0 & 0 & 1 & 3 \\
$4(1-6)$ & 0 & 0 & 1 & 0 & 0 & 1 \\
$5(2-3)$ & 1 & 0 & 0 & 0 & 1 & 2 \\
$6(2-4)$ & 1 & 0 & 0 & 1 & 1 & 3 \\
$7(2-6)$ & 1 & 1 & 1 & 0 & 1 & 4 \\
$8(3-5)$ & 1 & 1 & 0 & 1 & 0 & 3 \\
$9(3-6)$ & 1 & 0 & 1 & 0 & 1 & 3 \\
$10(4-6)$ & 1 & 1 & 0 & 0 & 1 & 3 \\
$11(5-6)$ & 1 & 1 & 1 & 0 & 0 & 3 \\
\hline$I$ & 36.900 & 24.167 & 17.747 & 7.938 & 16.759 & 103.51 \\
$G$ & 11529 & 13354 & 15606 & 17207 & 19479 & 77175 \\
$L$ & 46.990 & 1.241 & 5.6148 & 1.7241 & 3.4018 & 58.972 \\
$T$ & 11613 & 13380 & 15629 & 17216 & 19499 & 77337 \\
\hline
\end{tabular}

(b)

\begin{tabular}{ccccccc}
\hline $\begin{array}{c}\text { right-of- } \\
\text { way }\end{array}$ & Year 1 & Year 2 & Year 3 & Year 4 & Year 5 & Total \\
\hline $1(1-2)$ & 1 & 1 & 0 & 1 & 0 & 3 \\
$2(1-4)$ & 1 & 1 & 1 & 0 & 1 & 4 \\
$3(1-5)$ & 1 & 1 & 0 & 1 & 1 & 4 \\
$4(1-6)$ & 1 & 1 & 1 & 0 & 0 & 3 \\
$5(2-3)$ & 1 & 0 & 0 & 1 & 0 & 2 \\
$6(2-4)$ & 0 & 1 & 0 & 1 & 1 & 3 \\
$7(2-6)$ & 1 & 1 & 1 & 0 & 1 & 4 \\
$8(3-5)$ & 1 & 1 & 1 & 0 & 0 & 3 \\
$9(3-6)$ & 1 & 0 & 0 & 0 & 0 & 1 \\
$10(4-6)$ & 1 & 1 & 1 & 0 & 0 & 3 \\
$11(5-6)$ & 1 & 1 & 0 & 1 & 0 & 3 \\
\hline$I$ & 39.700 & 34.167 & 17.837 & 14.368 & 11.025 & 117.09 \\
$G$ & 11529 & 13354 & 15606 & 17207 & 19479 & 77174. \\
$L$ & 58.003 & 0.0000 & 0.0000 & 0.0000 & 0.0000 & 58.003 \\
$T$ & 11627 & 13388 & 15624 & 17221 & 19490 & 77349 \\
\hline
\end{tabular}

(c)

\begin{tabular}{ccccccc}
\hline $\begin{array}{c}\text { right-of- } \\
\text { way }\end{array}$ & Year 1 & Year 2 & Year 3 & Year 4 & Year 5 & Total \\
\hline $1(1-2)$ & 1 & 1 & 0 & 0 & 0 & 2 \\
$2(1-4)$ & 1 & 0 & 0 & 1 & 0 & 2 \\
$3(1-5)$ & 1 & 0 & 1 & 0 & 0 & 2 \\
$4(1-6)$ & 1 & 1 & 0 & 1 & 1 & 4 \\
$5(2-3)$ & 1 & 1 & 1 & 1 & 0 & 4 \\
$6(2-4)$ & 0 & 0 & 1 & 1 & 0 & 2 \\
$7(2-6)$ & 1 & 0 & 1 & 1 & 1 & 4 \\
$8(3-5)$ & 1 & 0 & 1 & 0 & 1 & 3 \\
$9(3-6)$ & 1 & 0 & 1 & 1 & 1 & 4 \\
$10(4-6)$ & 1 & 1 & 0 & 0 & 0 & 2 \\
$11(5-6)$ & 1 & 0 & 1 & 0 & 1 & 3 \\
\hline$I$ & 39.700 & 14.630 & 20.490 & 21.116 & 16.685 & 112.62 \\
$G$ & 11529 & 13354 & 15606 & 17207 & 19479 & 77174 \\
$L$ & 58.003 & 9.4612 & 0.3151 & 0.0000 & 0.0000 & 67.779 \\
$T$ & 11627 & 13378 & 15627 & 17228 & 19495 & 77355 \\
\hline
\end{tabular}

TABLE VI

PlanNing SChemes In the SElECted SubSET AND "Good ENOUGH" SUBSET (SUBSET G IS TOP $1 \%$ OF $\Theta^{N}$ )

\begin{tabular}{|c|c|}
\hline $\begin{array}{l}\text { Selected subset } \mathrm{S} \\
\quad(k=1, S=45)\end{array}$ & $\begin{array}{l}\mathbf{2 5 7}, \mathbf{9 9 4}, \mathbf{6 2}, \mathbf{4 2}, \mathbf{1 0}, \mathbf{5 3 9}, \mathbf{8 5 4}, \mathbf{8 6 5}, \mathbf{2 9 1}, \\
896,38,[\mathbf{9 7 3}, 361,944,99,768,30,551,111, \\
166,470,572,860,809,247,656,934, \mathbf{1 8 9}, \\
621,450,256,643,233,377,198,569,919, \\
442,332,683,878,13,322,565,476\end{array}$ \\
\hline $\begin{array}{l}\text { Good enough set } \mathrm{G} \\
(g=10)\end{array}$ & $\frac{865}{973}, 854,291,42,189,694,257,62,539$, \\
\hline
\end{tabular}

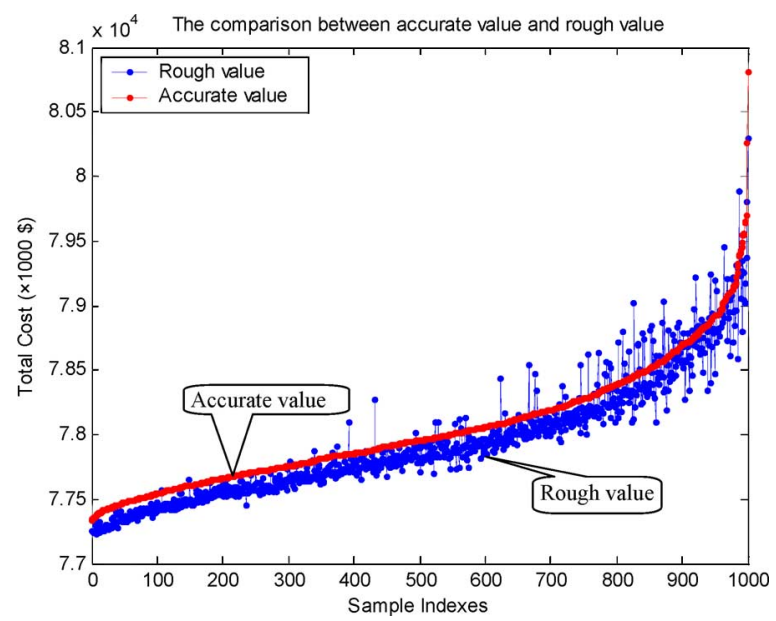

Fig. 3. Ordered performance curve for the Garver system.

It should be pointed out that though the OO theory assumes that errors are random, biased errors do not render the theory invalid. This is because the assumption concerning the error terms is used in two occasions. The first occasion is in the proof that the OPCs with or without errors (i.e., based on a crude or the exact models) belong to the same type (relative to the five types in Fig. 1). Clearly, biased errors would not change the shape of OPC either. The second occasion is when the error term is used in the selection of the size of the "selected subset S", where the "error bound" is one of the parameters in the selection formula. The error bound used in the theory was assumed to be "two-sided," whereas the biased error may be "one-sided." The use of a two-sided error bound in the formula in this case may be a little conservative (the size of $\mathrm{S}$ is unnecessarily large) when the errors are one-sided. In our example, errors seem to be biased. This may be part of the reason why our results are better (they capture all the good enough solutions, rather than just some of them) than the OO theory predicted.

The distribution of errors of the rough estimation is illustrated in Fig. 4. Comparing the exact results and rough estimations of the 1000 samples, we found that scheme no. 75 has the maximum error. The error of this scheme is listed in Table VII. The maximum error is only $0.94 \%$. The small errors between the crude model and the exact model may explain the reason why all elements in the selected subset in Table IV are from the good enough subset.

The normalized errors for $\mathrm{N}=1000$ samples have a standard deviation of $\sigma=0.0867$, which is in fact smaller than that of the 30 samples we used to determine subset size $s$.

The average time of exact computation of a sample scheme is about $1.04 \mathrm{~h}$. We used three computers to calculate the 1000 samples. It took about 15 days to obtain the exact results for all 1000 samples on three computers. However, the ordinal optimization approach takes only 2.12 hours on one computer. The computation time has been reduced to about $0.5 \%$. The computers used have a Pentium 4 microprocessor, a $2.4 \mathrm{GHz} \mathrm{CPU}$, and $512 \mathrm{MB}$ of memory.

\section{CONCLUSION}

Transmission expansion planning in the restructured industry is not standardized. However, its complexity makes simulation 


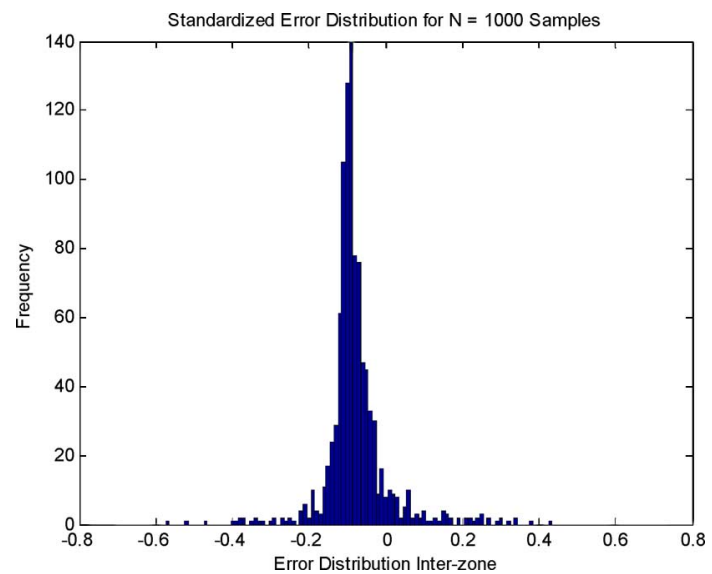

Fig. 4. Standardized error distribution for $N=1000$ sample

TABLE VII

ERROR OF SCHEME No. 75 (UNIT: 10 000\$)

\begin{tabular}{|c|c|c|c|c|}
\hline Scheme No. & Accurate cost & Rough cost & Error & Error $(\%)$ \\
\hline 75 & 79327.4 & 78579.5 & -747.9 & 0.94 \\
\hline
\end{tabular}

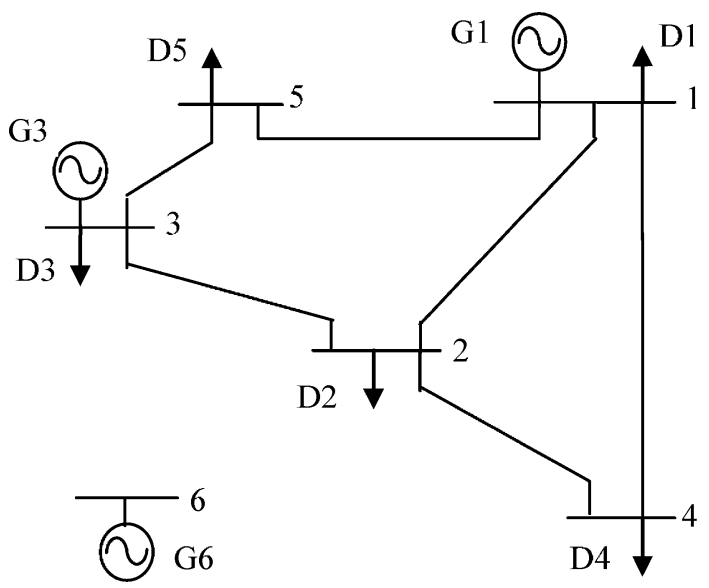

Fig. 5. Original six-node Garver system.

TABLE VIII

BASIC DATA FOR THE MODIFIED GARVER SYSTEM

\begin{tabular}{cccccc}
\hline $\begin{array}{c}\text { Right-of-way } \\
\text { (from-to) }\end{array}$ & $\begin{array}{c}\text { Reactance of } \\
\text { each line in the } \\
\text { right-of-way }\end{array}$ & $\begin{array}{c}\text { Original number of Capacity } \\
\text { lines in the right- } \\
\text { of-way }\end{array}$ & $\begin{array}{c}\text { Investment } \\
\text { of each } \\
\text { line (MW) }\end{array}$ \\
$\begin{array}{c}\text { for new line } \\
\left(10^{4} \$ / \text { line) }\right.\end{array}$ \\
\hline 1 (1-2) & 0.40 & 1 & 100 & 40 \\
2 & $(1-4)$ & 0.20 & 1 & 100 & 60 \\
3 & $(1-5)$ & 0.20 & 1 & 100 & 20 \\
4 & $(1-6)$ & 0.38 & 0 & 100 & 68 \\
5 & $(2-3)$ & 0.20 & 1 & 100 & 20 \\
6 & $(2-4)$ & 0.30 & 1 & 100 & 40 \\
7 & $(2-6)$ & 0.30 & 0 & 100 & 30 \\
8 & $(3-5)$ & 0.20 & 1 & 100 & 20 \\
9 & $(3-6)$ & 0.38 & 0 & 100 & 48 \\
$10(4-6)$ & 0.30 & 0 & 100 & 30 \\
$11(5-6)$ & 0.21 & 0 & 100 & 61 \\
\hline
\end{tabular}

the only viable approach to evaluate the performance of alternative planning schemes. We submit that ordinal optimization can be effectively applied for the simulation-based multiyear TEP problem. The goal of this paper is not to document how to apply
TABLE IX

QUADRATIC GENERATION COST FUNCTION (\$)

\begin{tabular}{ccc}
\hline Generator & Capacity (MW) & Cost function \\
\hline G1 & 600 & $0.01 \times g^{2}+20 \times g+150$ \\
G3 & 400 & $0.03 \times g^{2}+30 \times g+180$ \\
G6 & 800 & $0.02 \times g^{2}+25 \times g+100$ \\
\hline
\end{tabular}

TABLE $X$

QUADRATIC LOSS OF LOAD COST FUNCTION (\$)

\begin{tabular}{ccc}
\hline Load & Capacity (MW) & Cost function \\
\hline D1 & 0.1442 & $11.71 \times \Delta^{2}$ \\
D2 & 0.2885 & $3.9 \times \Delta^{2}$ \\
D3 & 0.1442 & $10.41 \times \Delta^{2}$ \\
D4 & 0.1923 & $7.32 \times \Delta^{2}$ \\
D5 & 0.2308 & $6.01 \times \Delta^{2}$ \\
\hline
\end{tabular}

the technique to a particular system. Rather, it is to demonstrate that the $\mathrm{OO}$ approach is effective in the sense that it is able to pick a "selected subset" in which enough "good enough" solutions can be found, and therefore simulations may be confined to the elements of a small selected subset. This was done based on the calculations made on a crude model. The classical TEP problem is used for demonstration. Furthermore, a brief and self-contained exposition of the ordinal optimization theory is presented. The approach can be adopted by planners with a proper selection of the crude and exact models of the specific TEP problem.

\section{APPENDIX}

The original six-node Garver system is shown in Fig. 5. Table VIII has the basic data for the modified Garver system, Table IX has the quadratic generation cost function (\$), and Table $\mathrm{X}$ has the quadratic loss of load cost function $(\$)$.

\section{REFERENCES}

[1] L. L. Garver, "Transmission network estimation using linear programming," IEEE Trans. Power App. Syst., vol. PAS-89, no. 7, pp. 1688-1679, Sep. 1970

[2] R. Villasana, L. L. Garver, and S. J. Salon, "Transmission network planning using linear programming," IEEE Trans. Power App. Syst., vol. PAS-104, no. 2, pp. 349-356, Feb. 1985

[3] A. P. Meliopoulos, R. P. Webb, R. J. Bennon, and J. A. Juves, "Optimal long-range transmission planning with ac load flow," IEEE Trans. Power App. Syst., vol. PAS-101, no. 10, pp. 4156-4163, 1982.

[4] Z. M. Al-Hamouz and A. S. Al-Faraj, "Transmission expansion planning using nonlinear programming," in Proc. 2002 IEEE Power Eng. Soc. Transmission and Distribution Conf., 2002, vol. 1, pp. 50-55.

[5] N. Alguacil, A. L. Motto, and A. J. Conejo, "Transmission expansion planning: A mixed-integer LP approach," IEEE Trans. Power Syst., vol. 8, no. 3, pp. 1070-1077, Aug. 2003.

[6] L. Bahiense, G. C. Oliveira, M. Pereira, and S. Granville, "A mixed integer disjunctive model for transmission network expansion," IEEE Trans. Power Syst., vol. 16, no. 3, pp. 560-565, Aug. 2001.

[7] A. Santos, P. M. Franca, and A. Said, "An optimization model for longrange transmission expansion planning," IEEE Trans. Power Syst., vol. 4, no. 1, pp. 94-101, Feb. 1989.

[8] A. Seifu, S. Salon, and G. List, "Optimization of transmission line planning including security constraints," IEEE Trans. Power Syst., vol. 4, pp. 1507-1512, Nov. 1989

[9] S. Binato, M. V. F. Pereira, and S. Granville, "A new Benders decomposition approach to solve power transmission network design problems," IEEE Trans. Power Syst., vol. 16, no. 2, pp. 235-240, May 2001. 
[10] S. N. Siddiqi and M. L. Baughman, "Value-based transmission planning and the effects of network models," IEEE Trans. Power Syst., vol. 10, no. 4, pp. 1835-1842, Nov. 1995.

[11] G. C. Oliveira, A. P. C. Costa, and S. Binato, "Large scale transmission network planning using optimization and heuristic techniques," IEEE Trans. Power Syst., vol. 10, no. 4, pp. 1828-1833, Nov. 1995.

[12] C. W. Lee, K. K. Ng, J. Zhong, and F. F. Wu, "Transmission expansion planning from past to future," in Proc. IEEE/Power Eng. Soc. Power System Conf. Expo., Atlanta, GA, Oct. 2006.

[13] The California ISO and London Economics International LLC, Tech. Rep., A Proposed Methodology for Evaluating the Economic Benefits of Transmission Expansions in a Restructured Wholesale Electricity Market, Feb. 2003. [Online]. Available: http://www.caiso.com/docs/ 2003/03/18/2003031815303519270.html.

[14] F. F. Wu, F. L. Zheng, and F. S. Wen, "Transmission investment and expansion planning in a restructured electricity market," Energy, vol. 31, pp. 954-966, 2006.

[15] Y. C. Ho, "An explanation of ordinal optimization: Soft computing for hard problems," Inform. Sci., vol. 113, pp. 169-192, 1999.

[16] D. Conforti, D. Menniti, and N. Sorrentino et al., "Optimal load-flow with $\mathrm{N}-1$ steady-state security via high performance computing," in Proc. Mediterranean Electrotechnical Conf.-MELECON, Industrial Applications in Power Systems, Computer Science and Telecommunications, 1996, vol. 2, pp. 873-879.

[17] T. W. E. Lau and Y. C. Ho, "Universal alignment probabilities and subset selection for ordinal optimization," J. Optim. Theory Appl., vol. 93, no. 3, pp. 445-489, Jun. 1997.

[18] Y. C. Ho, R. S. Sreenivas, and P. Vakili, "Ordinal optimization in DEDS," J. Discrete Event Dyn. Syst., vol. 2, pp. 61-68, 1992.

[19] Y. C. Ho, "Overview of ordinal optimization," in Proc. 33rd IEEE Conf. Decision and Control, Lake Buena Vista, FL, 1994, pp. 1975-1977.

[20] L. Dai, "Convergence properties of ordinal comparison in the simulation of discrete event dynamic systems," J. Optim. Theory Appl., vol. 91, pp. 363-388, 1996.

[21] D. Li, L. H. Lee, and Y. C. Ho, "Constrained ordinal optimization," Inform. Sci., vol. 148, pp. 201-220, 2002.

[22] D. Li, L. H. Lee, and Y. C. Ho, "Vector ordinal optimization: A new heuristic approach and its application to computer network routing design problems," Int. J. Oper. Quant. Manage., vol. 5, pp. 211-230, 1999.
[23] Y. C. Ho and M. Larson, "Ordinal optimization and rare event probability simulation," J. Discrete Event Dyn. Syst., vol. 5, no. 2-3, pp. 281-301, 1995.

[24] Y. C. Ho, Soft Optimization for Hard Problems. [Online]. Available: www.hrl.harvard.edu/people/faculty/ho/DEDS. Oct. 1997.

[25] M. Deng and Y. C. Ho, "Sampling selection method for stochastic optimization problems," Automation, vol. 35, no. 2, pp. 331-338, 1999.

[26] S.-Y. Lin, Y.-C. Ho, and C.-H. Lin, "An ordinal optimization theorybased algorithm for solving the optimal power flow problem with discrete control variables," IEEE Trans. Power Syst., vol. 19, no. 1, pp. 276-286, Feb. 2004

[27] X. Guan, Y.-C. Ho, and F. Lai, "An ordinal optimization based bidding strategy for electric power suppliers in the daily energy market," IEEE Trans. Power Syst., vol. 16, no. 4, pp. 788-797, Nov. 2001.

Min Xie received the B.Sc (Eng.) degree from the Central South College of Science and Technology, Hunan, China, in 2000 and the Ph.D. degree from the Huazhong University of Science and Technology, Wuhan, China, in 2006.

She is currently a Lecturer with the School of Electric Power at the South China University of Technology. Her area of interest is power system planning in deregulated power system.

Jin Zhong (S'00-M'04) received the B.Sc. (Eng.) degree from Tsinghua University, Beijing, China, in 1995, the M.Sc. (Eng.) degree from China Electric Power Research Institute (CEPRI), Beijing, in 1998 and the Ph.D. degree from Chalmers University of Technology, Gothenburg, Sweden, in 2003.

She was a Researcher with CEPRI in 1998-1999. Currently, she is an Assistant Professor with the Department of Electrical and Electronic Engineering, University of Hong Kong, Hong Kong, China.

Felix Wu (M'74-F'89) is the Philip Wong Wilson Wong Professor in Electrical Engineering at the University of Hong Kong, Hong Kong, China, where he served as Vice President from 1997 to 2001. He is also a Professor Emeritus at the University of California, Berkeley, where he has been on the faculty since 1974. 